\title{
Securing Helwan University Graduation Temporary Certificate against the risks of forgery and counterfeiting by using security features
}

\author{
Mahmoud Farouk El Feky \\ Assistant Professor at Printing, Publishing and Packaging Department, \\ Faculty of Applied Arts - Helwan University, Egypt \\ e-mail: drmahmoudelfeky@ gmail.com
}

\begin{abstract}
"Helwan University graduation temporary certificate" is a very valuable authentic document, like any graduation temporary certificate all over the universities. It is issued for graduates who need it to present to everyone or firm who is interested. However, it is not secured with any security feature that prevent forgery, tampering or altering the information of the certificate by using any professional image processor application like Photoshop. So there was an urgent need to introduce an innovative design that achieves two goals, first of all is securing the certificate against counterfeiting and forgery, secondly, creating an aesthetic appeal to it. So the research hypothesis was that creating an innovative decorative design supported with some suggested security features like "Guilloché", "Micro-printing", "Optically Variable Inks (OVI)" and printed "Radio frequency Identification (RFID) tag", will definitely achieve these goals, provided do not considerably increase the cost of the produced documents. After experimental work study, the results shown that this innovative design were generated by Adobe Illustrator CC 2015 software, has achieved the research goals.
\end{abstract}

\section{Keywords}

Forgery and counterfeiting, Security features, Graduation temporary certificate, Guilloche, Microprinting, Optically Variable Ink, OVI, Radio Frequency Identification System, RFID tag

\section{Introduction}

Forgery is making alterations to a genuine document. It is a tricky problem because it deals with an authentic document in which the security features are still present. For example, "successful alteration of a document leaves the bulk of the genuine authenticity features unaltered and facilitates acceptance of the document by authorities" (Corbin Nakamura, 2010).

Forgery of certificates is becoming now more than ever a serious problem all over the world, due to the availability of high quality and low-priced color photocopiers and desktop publishing systems, also availability of very talented designers who uses image processing applications. The main goal of security printing is to prevent forgery, tampering, or counterfeiting. In recent days, the industry of security printing is struggling to bring up new ideas, with more security features and less coasts to protect documents from counterfeiting or alteration of information (forgery). (Iskren et al, 2018), some of the already used 
anti-counterfeit and authentication means include the use of special paper, special inks, watermarks, micro-letters, security threads, holograms, etc. Nevertheless, there is still an urgent need to introduce further security elements, which do not considerably increase the cost of the produced documents. (Aryachandran S. and Jyothi R. L., 2014), In our case, "Helwan University graduation temporary certificate" is a very valuable authentic document that is issued for graduates who need it to present to everyone or firm who is interested. However, it is not secured with any security feature that prevent forgery, tampering or altering the information of the certificate by using any professional image processor application like Photoshop, as shown in (figure 1), In response to this need, I was assigned by "The faculty of Applied Arts Vice Dean for Education and Student Affairs" to make an innovative design for the "Helwan University graduation temporary certificate" that achieves two goals, first of all is securing the certificate against counterfeiting and forgery, secondly, creating an aesthetic appeal to it.

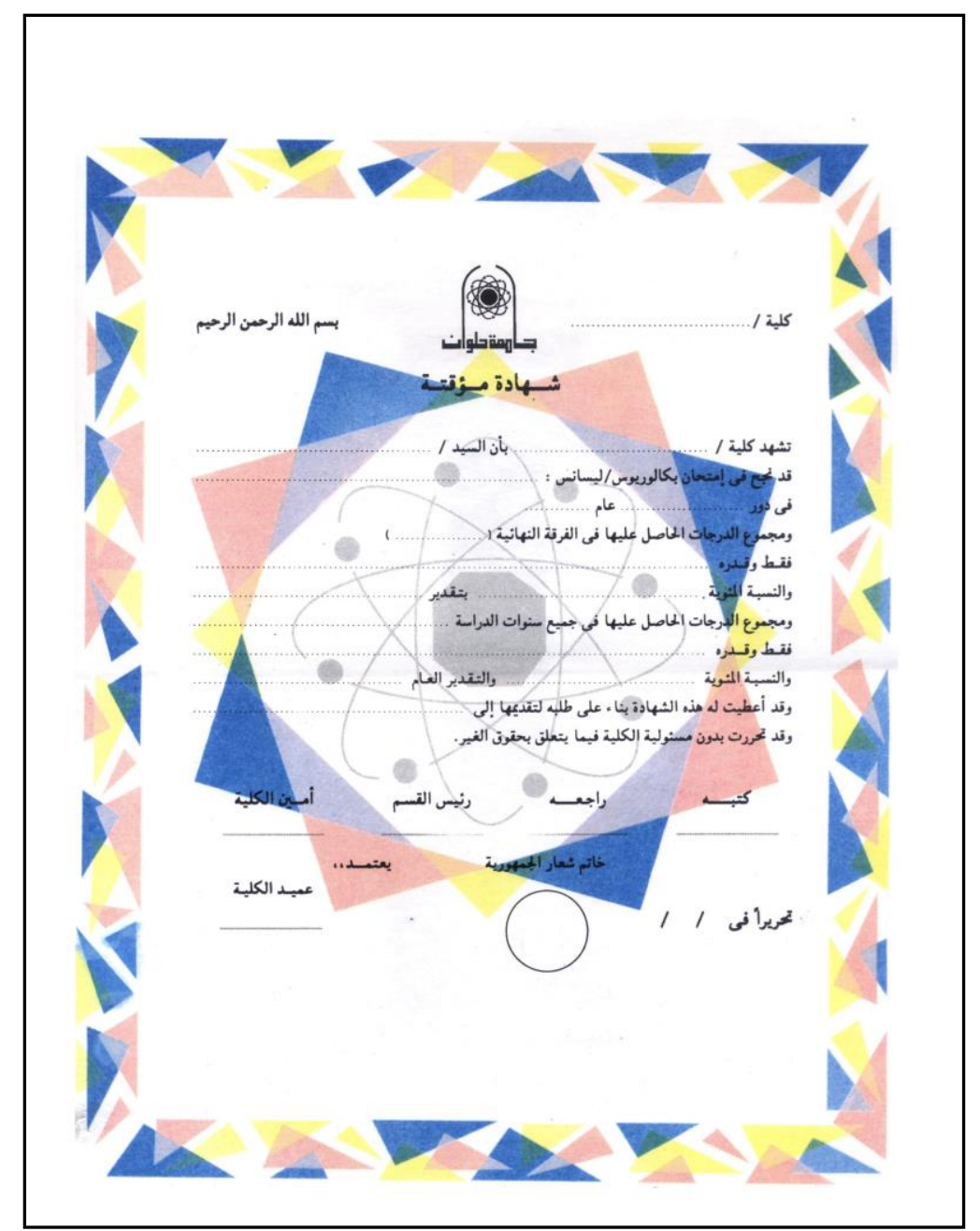

Fig 1: illustrate The old design of Helwan University Certificate

\section{Originality/ value:}

This study serves in adding an aesthetic appeal to the graduation certificate "Helwan University graduation temporary certificate", preventing the forgers 
from alternating the certificate information (forgery), protecting the authentic certificate holder rights, and simultaneously protecting the university rights from scammers.

\section{Literature Review:}

Security printing features are often categorized as either "overt" or "covert". Overt security features are integrated into documents and are designed to be easily detectable by the unaided eye under normal daylight or office lighting. Covert devices are usually hidden in the document's design. They can be machine-readable or require special Optical aids to be detected and verified. (T. Bozhkova et al, 2017)

There are many features, which used for security and they get involved in different steps production, i.e. security involved at substrate level when it is manufactured, the designing elements which are involved in document for security, the printing processes and special applications used and at last different post press special applications used. The contents of security printing which are incorporated in document security are differentiated on the basis of where and how these involved and added. (Parag Dnyandeo, 2012)

- One of these designing elements is "Guilloché", it is a unique standard overt feature which are designed to be hard to reproduce, to protect documents. It is used as background patterns on virtually all currency notes, passports or any authentication documents. (Zhigang Fan, et al 2008), Using a guilloche pattern as a background can be very effective to protect a document from counterfeiters. (T. Bozhkova, 2017)

- Another important designing element is "Microprinting", it is a covert security feature that makes it harder to fake official documents and print it. Microprinting is the printing of extremely fine details on a document. When used as an antifraud tool, small letters are often printed so small that they the human eye cannot read them; they can become visible as lines with the unaided eyes. Under the magnification it can be clearly read. More importantly, microprinting helps you verify when a document is legitimate. (Justin Pritchard, 2019) Micro printed features are hard to scan. (Anju Annie Thomas, et al, 2018). Therefore, when microprint was first used, very few copiers or scanners could copy the small letters with sufficient resolution, so those tools would produce an easy-to-detect fake. Even with a powerful scanner, you'd need a very fancy printer to clearly print tiny letters. These hurdles make it hard for anybody (except the most dedicated and best-equipped scammers) to create genuine-looking fakes, although it is getting easier as printers and scanners improve. (Justin Pritchard, 2019)

- Another important designing element is "Optically Variable Inks (OVI)", it is a special kind of ink used as marker in important documents as an anticounterfeiting measure. Depending on the angle of view, the ink shows two colors generally, orange, blue, green etc. The $O V I$ is highly useful to show the authenticity of the documents since the use of the ink is strictly controlled and not available easily. 
OVI contain tiny flakes of special film which changes color as the viewing angle is varied. $O V I$ is a very expensive ink and generally used only in small areas of the document to mark emblems, identity marks etc. Screen printing method is sometime used to make $O V I$ documents. It is widely used to make marks in currencies.

Depending on the angle of incidence of light, the $O V I$ reflects different wavelengths of white light. As a result, a color change feels when viewed with unaided eye. If the document is copied using a scanner, only one color will appear in the image so that it is easy to check the documents with the original one. $O V I$ is considered as the most complicated type of ink to prevent forgery of documents. The ink has a colorful metal luster with two colors. Various combinations of colors are Red-Green, Gold-Silver, Green-Blue combinations. When viewed under sunlight, the ink gives two colors with optical variation and sharp color change. Due to its complicated ink production procedure, huge investment, high anti-forgery reliability, it has become an excellent anti-forgery ink. Moreover, the ink is highly resistant to environmental factors such as sunlight, moisture etc. (D. Mohankumar, 2010)

- Finally, an important designing element is "Radio frequency Identification (RFID), it is not a new technology. RFID Systems consist of a transponder, also known as a tag, which is basically a microchip connected to an antenna. The tag is mounted to an item, such as our certificate, and a device called a reader communicates with the tag via radio waves. Depending on the type of tag that is used, the reader can receive detailed information or it can receive data as simple as an identification number.

The basic types of RFID tags can be classified as read/write and read only. Also they are further categorized as: Active tags, Semi-active (or semi-passive) tags, or Passive tags, in our certificate the type is printed "Read/Write Passive RFID tag", components of passive RFID system are:

- An antenna is attached to a microchip.

- The antenna allows the chip to transmit information to a reader, which also has an antenna.

- The reader is the device that actually sends out the radio waves to create a magnetic field. A passive RFID tag draws its power from this magnetic field, which powers the circuits in the microchip allowing it to transmit data back to the reader.

- Reader transmits to a computer system.

- The computer passes data onto a network.

- Software determines how the data received should be used.

With the overgrowing needs of greater security features, RFID tags can be evolved in taking care for the high security of authentication documents like graduation certificates.

These passive RFID tags (shown on Figure 2) are much cheaper and can be preordered and attached as a hidden label on the certificate already printed sheet of paper, or in search for a greater security, they can be ordered built in the paper, ready to print over. 
After having the certificates printed and with an $R F I D$ tag already built in (Figure 3), the further need is only to prepare the RFID tag reader. This can automatically prevent the needs of eye checks for watermarks and other common security features for high end security products that can be manipulated easily. (Iskren et al, 2018)

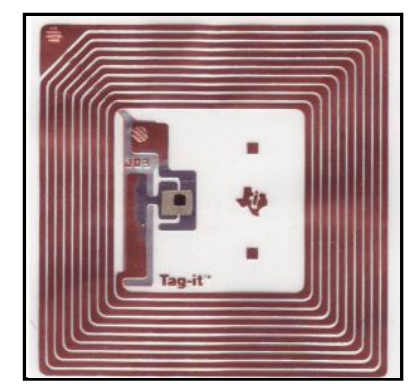

Fig 2: illustrate The Passive RFID tag

\section{Objectives}

The main goal in this study is to secure "Helwan University graduation temporary certificate" from forgery, tampering, or counterfeiting. And in the same time, to add an aesthetic appeal to it.

\section{Methodology}

In order to achieve the research objectives, an experimental study is conducted as following:

\section{Materials and procedures:}

A new innovative design structured with some security features like "Guilloché" patterns, "Microprinting" patterns, Optically Variable Ink (OVI), and "RFID tag" were generated by Adobe Illustrator CC 2015 software, and these features were designed as following:

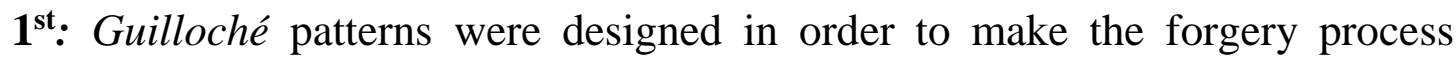
difficult, they are located as following:

- The whole background, as shown in (figure 3)

- The University logo in the center of the certificate, as shown in (figure 4)

- The repeated motif in the certificate bottom, as shown in (figure 5) and the motif covers the hidden printed passive RFID tag, which located at the bottom right of the certificate, as shown in (figure 6)

$2^{\text {nd }}$ : Microprinting patterns were designed resulting in extremely tiny words located in particular places in order to make the forgery process impossible, they can become visible as lines with the unaided eyes. But under the magnification they can be clearly seen as the name of the university in Arabic "جامعة حلوان", they are located as following:

- The 2 circles surrounding the university logo in the center of the certificate, as shown in (figure 7) 
- The circle designed as guide to print the "Eagle Stamp" at the bottom of the certificate, as shown in (figure 8)

$3^{\text {rd: }}$ Outline University logo pattern were repeated in combining with guilloche pattern in the background in particular places in order to make the forgery process difficult, as shown in (figure 9)

$4^{\text {th }}$ : A motif of guilloche printed with an Optically Variable Ink (OVI), as shown in (figure 10)

$5^{\text {th }}$ : Hidden printed RFID passive tag, which located at the bottom right of the certificate, as shown in (figure 11),

$\mathbf{6}^{\text {th }}$ : the author advised to print the certificate on a special secured paper, which contains covert security features involved at the substrate when it is manufactured, like watermark, fluorescent fibers etc.

Finally, Helwan University accepted another suggested design, as shown in (figure 12). The selected design contains only an overt security feature, "Guilloche", in some places like background, frame and another objects, as shown in (figure 13), covert security feature, "Microprinting", in the center of the frame, as shown in (figure 14), and finally covert security features involved at substrate level when it is manufactured, like "CBE" watermark and fluorescent fibers. 
Fig 3: illustrates The guilloche pattern in the whole background

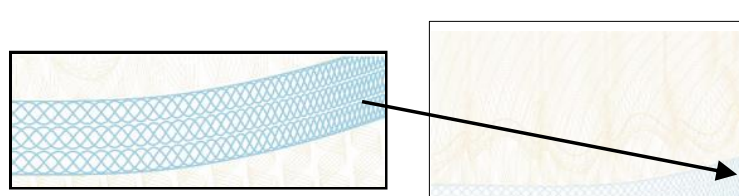

Fig 10: illustrates The guilloche motif printed with Optically Variable Ink

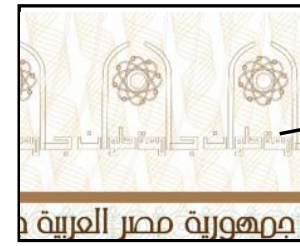

Fig 9: illustrates The Outline

University logo pattern

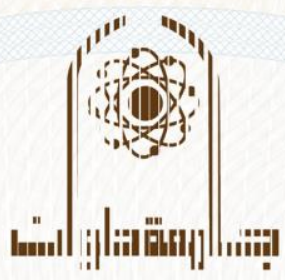

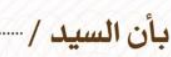

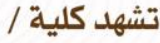

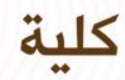

قد نجح في إمتحان بكالوريوس/ليسانس: /...

عام

في دور - مجوع

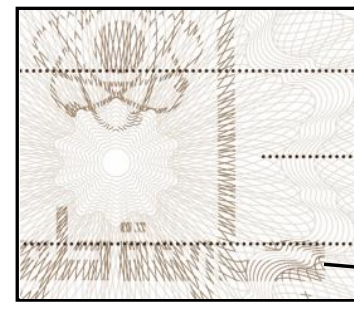

مجموع الدرجات الحاصل عليها في الفرقة النهائية ( مقاية

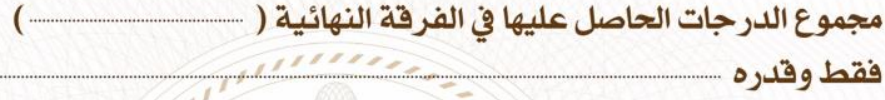

Fig 4: illustrates The University logo in the center of the certificate

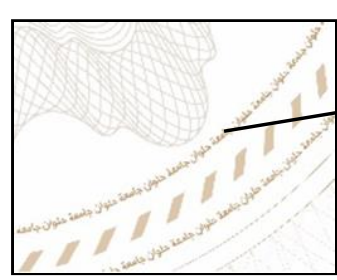

Fig 7: illustrates microprinting in The 2 circles surrounding

the university logo

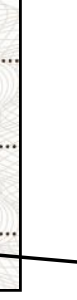
بتقدير النسبة المئوية

مجموع الدرجات الحاصل عليها في جميع سنوات الدراسة فقط وقدره النسبة المئوية مشروع التخرج

وقد أعطيت له هذه الشهادة بناء على طلبه لتقديمها إلى وقد تحررت بلدون مسئولية الكلية فيما يتعلق بحقوق الغير.

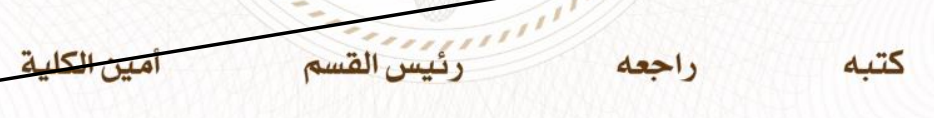

يعتمل،،

خاتم شعار الجمهورية

عميد الكلية

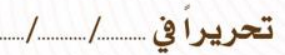

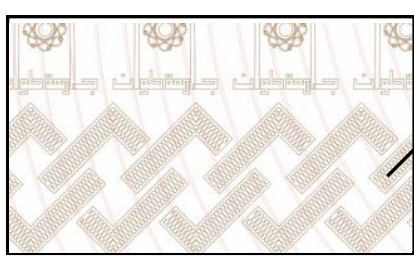

Fig 5: illustrates the repeated motif in the certificate bottom
Fig 8: illustrates microprinting in The circle designed as guide to print the "Eagle Stamp"

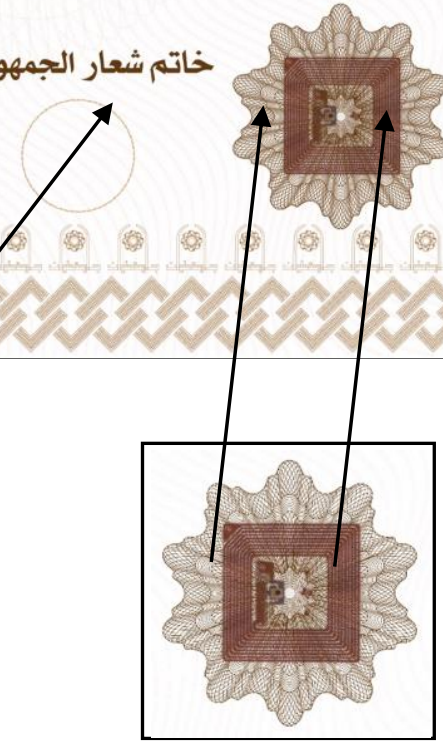

Fig 6,11: illustrate Hidden RFID tag under the guilloche motif 


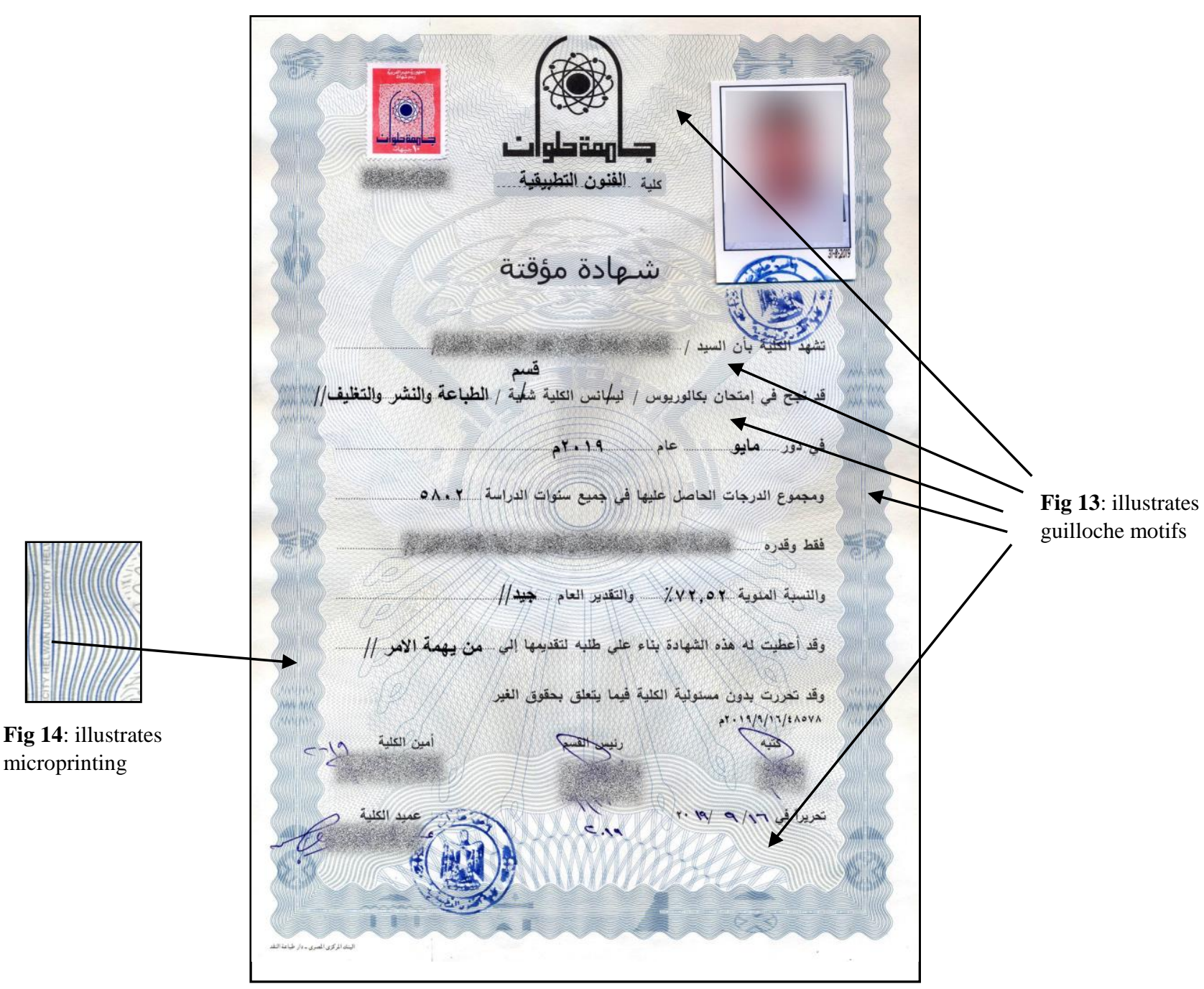

Fig 12: illustrates the final selected design (current design)

\section{Results:}

There are three different results for the research experimental procedures:

$\mathbf{1}^{\text {st. }}$ As shown in figure 1 , the old design of the certificate is very poor aesthetically and not safe, so it was necessary to change the design.

$2^{\text {nd: }}$ The author suggested design is an innovative design, which includes many items that add an aesthetic appeal to the certificate, and help in preventing forgery, tampering, or counterfeiting by scammers, moreover make it impossible, as shown in figures from 3 to 11 .

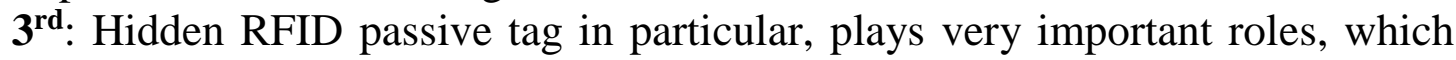
stores the personal information of the authentic certificate holder, so it is an identification system and in the same time plays as a very important security feature which protect the authentic certificate holder rights.

$4^{\text {th: }}$ : The final selected design is different from the old one, but it has not enough security features when compared to the author suggested design. 


\section{Conclusion}

As a result of the investigation, especially when we talk about very important valuable authentic document like "Helwan University graduation temporary certificate", which needs to be secured from alternating its information, protecting the authentic certificate holder rights, and simultaneously protecting the university rights from scammers, it was very important to make an innovative design that achieves two goals, first of all is securing the certificate against counterfeiting and forgery, secondly, creating an aesthetic appeal to it. The design structured with some security features like "Guilloché" patterns, "Microprinting" patterns, Optically Variable Ink (OVI), and "RFID tag" were generated by Adobe Illustrator CC 2015 software, in order to achieve the research goals.

\section{References}

1- Anju Annie Thomas, Emna Jeridi, Bhoopesh Kumar Sharma, Ved P. Mishra, Mohamamad Al Shamsi, Mohammad Al Khalloufi, (2018), Study of Security Features of Bank Cheques and Credit Cards and Decipherment, International Conference on Reliability, Infocom Technologies and Optimization (ICRITO) (Trends and Future Directions), pp 209

2- Aryachandran S. and Jyothi R. L., (2014), Secure Color QR Codes, IOSR Journal of Computer Engineering (IOSR-JCE), National Conference in Emerging Technologies, pp78

3- Corbin Nakamura, (2010), The Security Printing Practices of Banknotes, the Faculty of the Graphic Communication California Polytechnic State University, San Luis Obispo, pp12,19

4- D. Mohankumar, (2010), Optically Variable Ink, Mohan's electronics blog

5- Justin Pritchard, (2019), What Is Microprinting on a Check?

6- Iskren Spiridonov, Kosta Shterev and Tatyana Bozhkova, (2018), Future Development of Security Printing and RFID Marks, the University of Novi Sad, Faculty of Technical Sciences, Department of Graphic Engineering and Design, pp71,72

7- Parag Dnyandeo Nathe, (2012), Analysis of security printing features accomplished by sheetfed lithographic offset process \& sheetfed screen printing process, International Journal of Engineering and Management Sciences, VOL 3 (3), pp 256

8- T. Bozhkova, I. Spiridonov, K. Shterev (2017), Overview of security printing types and trends in its future development Bulgarian Chemical Communications, Volume 49, Special issue L (pp. 195 -201), pp197

9- Zhigang Fan, Reiner Eschbach, Shen-ge Wang, and Raja Bala, (2008), Security Printing in Digital Age, Xerox Corporation; Webster, NY, USA, ICISH: International Conference on Imaging Science and Hardcopy, pp 8.9 